\title{
Herpes Zoster Knowledge, Prevalence, and Vaccination Rate by Race
}

\author{
Tae Joon Lee, MD, CMD, Stella Hayes, MD, Doyle M. Cummings, PharmD, \\ Qing Cao, MD, Kristin Carpenter, Leah Heim, and Hollie Edwards
}

Objectives: To determine the prevalence of self-reported herpes zoster (HZ) disease and vaccination in a geriatric population and to characterize the deciding factors to receive the $\mathrm{HZ}$ vaccine.

Methods: This was a cross-sectional survey of patients older than age 60 years at 3 university-based primary care clinics in the southeastern United States. Participants provided information for age, race, sex, education level, history of having "shingles" or knowing someone else who had shingles, past vaccinations, and factors influencing their decision to receive the $\mathrm{HZ}$ vaccine.

Results: We surveyed 403 patients (49\% African American [AA], 47\% white). The prevalence of $\mathrm{HZ}$ was $12.4 \%$ overall and was significantly different among races $(8 \% \mathrm{AA}, 17 \%$ white; $P=.01)$. Only $29 \%$ of patients $(16 \% \mathrm{AA}, 42 \%$ white; $P<.001)$ were aware that the $\mathrm{HZ}$ vaccine was recommended. The $\mathrm{HZ}$ vaccination rate was $7.7 \%(2 \% \mathrm{AA}, 14 \%$ white; $P<.001)$. Only $13.7 \%$ of all study subjects reported having any communication with their medical providers regarding the $\mathrm{HZ}$ vaccine. Physician recommendation and media had the greatest influence on patients who received the vaccine. Of those who had not been vaccinated, $70 \%$ had never heard about it and 59\% were interested in receiving the vaccine after the survey.

Conclusion: There is a large difference in self-reported $\mathrm{HZ}$ and vaccination rates among races. The $\mathrm{HZ}$ vaccination rate was low overall, but most patients were interested in receiving the vaccine after the survey. More public awareness and education is needed to improve rates of $\mathrm{HZ}$ vaccination. ( $\mathrm{J}$ Am Board Fam Med 2013;26:45-51.)

Keywords: Elderly, Health Services for the Aged, Herpes Zoster, Immunization, Public Health, Vaccination

Herpes Zoster (HZ), also known as shingles, is a skin condition caused by the reactivation of a latent varicella zoster virus, which is the virus that also causes chickenpox. ${ }^{1} \mathrm{HZ}$ is most commonly seen in patients who are older than age 50, immunocompromised, or receiving immunosuppressive therapy. ${ }^{2}$ Every year, there are approximately 1 million new cases of $\mathrm{HZ}$ in the United States. ${ }^{3,4}$ In addition, the incidence of $\mathrm{HZ}$ varies among races.

This article was externally peer reviewed.

Submitted 6 June 2012; revised 17 August 2012; accepted 27 August 2012.

From the Geriatrics and Research Divisions, Department of Family Medicine, East Carolina University, Greenville, NC.

Funding: none.

Prior presentation: This work was presented as a poster at the annual meeting of the American Geriatrics Society, National Harbor, Maryland, May 2011.

Conflict of interest: none declared.

Corresponding author: Tae Joon Lee, MD, CMD, 101 Heart Drive, Greenville, NC 27834 (E-mail: leetae@ecu. edu).
There is a significantly higher rate of $\mathrm{HZ}$ among whites compared with African Americans and Hispanics. ${ }^{5-7}$ One major complication with $\mathrm{HZ}$ is postherpetic neuralgia, which is dermatomal pain after the resolution of rash that can be exquisitely painful and last months to even years. ${ }^{8,9}$

In 2008, the Advisory Committee on Immunization Practices recommended that all people older than age 60 receive the zoster vaccination. ${ }^{10}$ The vaccine is recommended without serologic testing and regardless of race or medical history of varicella virus infection or $\mathrm{HZ}$. When vaccinated, the risk of having $\mathrm{HZ}$, the burden of disease, and the incidence of postherpetic neuralgia decrease by $51 \%$, $61 \%$, and $66 \%$, respectively, over 3 years. ${ }^{2,10}$ In 2011, the US Food and Drug Administration approved $\mathrm{HZ}$ vaccination for immunocompetent people older than age $50 .{ }^{11,12}$

However, despite the recommendation of the Advisory Committee on Immunization Practices, 
the rate of zoster vaccination is low. Several studies have examined the rate of use of the $\mathrm{HZ}$ vaccine in a population. In 2007, approximately 3500 adults older than age 60 in the United States were surveyed, and $1.9 \%$ of those surveyed reported having had the $\mathrm{HZ}$ vaccine. ${ }^{13}$ Of those surveyed who had not been vaccinated, approximately $80 \%$ reported that they would receive the vaccine if their doctor recommended it. The 2 most common reasons for declining to be vaccinated were that the patients did not feel it was needed, and many did not think they were at risk. In 2009, the rate of zoster vaccination among a group of rheumatologic patients was still relatively low $(9.1 \%) .{ }^{14}$ One study reported that the major barrier to receiving the $\mathrm{HZ}$ vaccine was the cost. ${ }^{15}$ Of the vaccines recommended for the older population, the $\mathrm{HZ}$ vaccine is among the most expensive (approximately $\$ 150$ per dose). ${ }^{12,16}$

The purpose of this study was to determine the prevalence of $\mathrm{HZ}$ and the rate of vaccination among older patients now that the vaccine has been available and recommended for several years. We hypothesized that many of the patients who meet guideline recommendations to receive the $\mathrm{HZ}$ vaccine still will not have been vaccinated. We also were interested in any differences in the vaccination rate related to patient race, sex, education level, history of $\mathrm{HZ}$, and witnessing someone with $\mathrm{HZ}$, as well as reasons that influenced people's decisions regarding $\mathrm{HZ}$ vaccination. Once barriers are delineated, efforts can be made to improve $\mathrm{HZ}$ vaccination rates among appropriate patients.

\section{Methods}

This was a cross-sectional study of adult patients, aged 60 years and older, who were being seen in one of the 3 primary care practices in an academic medical center in eastern North Carolina. During the months of June and July 2010, the research team members visited these academic primary care practices and, using a questionnaire developed by the investigators, quickly collected study data from eligible patients waiting for their visit. The patients provided informed consent or had a family member or health care agent present to provide informed consent. Surveys were administered one-on-one in private examination rooms and, including the informed consent process, took approximately $5 \mathrm{~min}$ utes to complete. New patients to the clinic within the past 6 months were excluded to reduce outside influences on vaccination practices. Each study investigator conducted interviews at all the 3 primary care practices to minimize any investigator/practice bias. Data collected did not contain protected health information or patient identifiers and the study was approved by the University and Medical Center Institutional Review Board at East Carolina University.

The survey included questions concerning age, race, sex, educational level, vaccination history (including influenza, pneumococcus, and HZ), personal history of shingles, and witnessing someone with shingles. Depending on the respondents' answers regarding the vaccination history, additional questions were asked regarding reasons for and against receiving $\mathrm{HZ}$ vaccination, history of physician's or other provider's recommendation regarding $\mathrm{HZ}$ vaccination, and perceptions regarding the efficacy of the vaccination. The patient survey data were entered into Qualtrics (version 10.875), a computerized database, and was analyzed using PASW (formerly SPSS, version 17, IBM, Chicago, IL). $\chi^{2}$ analysis was used to examine differences in the prevalence of $\mathrm{HZ}$ and vaccination rates by race (African American vs white), sex (men vs women), and educational status (less than high school vs high school or higher education). Multivariable logistic regression analysis was used to examine $\mathrm{HZ}$ vaccination rates by race while controlling for age, sex, educational level, and witnessing someone with shingles.

\section{Results}

Our sample of 403 participants consisted of 132 men $(33 \%)$ and 271 women aged 60 to 97 years (mean age, 72.8 years). The racial composition was 198 African Americans (AAs) (49\%), 191 whites (47\%), 4 Asians, 4 Hispanics, 3 American Indians, and 1 "other." The average reported education level was through the 11 th grade, and $43 \%$ did not complete high school. Of the patients surveyed, 401 completed the entire survey and 2 partially completed the survey. The available portions of the 2 incomplete surveys were analyzed. Table 1 summarizes the demographic data of the study population.

Significant differences among African Americans and whites were noted in multiple study outcomes. The overall $\mathrm{HZ}$ vaccination rate was $7.7 \%$ 
Table 1. Participant Demographics $(\mathrm{N}=403)$

\begin{tabular}{lc}
\hline Mean age, years* & 72.8 \\
Sex & \\
Men & $132(33)$ \\
Women & $271(67)$ \\
Race & \\
African American & $198(49)$ \\
White & $191(47)$ \\
Asian & $4(1)$ \\
Hispanic & $4(1)$ \\
American Indian & $3(0.7)$ \\
Other & $1(0.2)$ \\
Mean education $\dagger$ & 11 th grade \\
Patient enrollment sites at East Carolina & \\
$\quad$ University & \\
Internal medicine clinic & $134(33)$ \\
Family medicine clinic & $133(33)$ \\
Geriatric clinic & $134(33)$ \\
\hline
\end{tabular}

*Range, 60-97 years.

†Range, 1-18 years.

( $2 \%$ AA, $14 \%$ white; $P<.001$ ). Self-reported prevalence of $\mathrm{HZ}$ was $12.4 \%$ overall ( $8 \% \mathrm{AA}, 17 \%$ white; $P=.01$ ). Of our participants, $52.6 \%$ had seen someone who had developed shingles, but that varied similarly between the races (35\% AA, 72\% white; $P<.001)$. Awareness that the $\mathrm{HZ}$ vaccine was recommended for all Americans older than 60 years was $29 \%$ ( $16 \%$ AA, $42 \%$ white; $P<.001)$. Table 2 shows the differences in the prevalence of self-reported HZ and other factors overall for African Americans and whites.

Although having a personal history of $\mathrm{HZ}$ was not associated with a higher rate of self-reported $\mathrm{HZ}$ vaccination $(12.9 \%$ vs $11.8 \% ; P=.87)$, witnessing someone with shingles was associated with a higher rate $\mathrm{HZ}$ vaccination compared with people who had never seen others with shingles (11.8\% vs $3.2 \% ; P<.001)$. Patients who reported receiving the $\mathrm{HZ}$ vaccine had a trend toward a higher self-reported rate of receiving the seasonal flu shot (97\%) compared with $88 \%$ of those who reported not receiving the $\mathrm{HZ}$ vaccine $(P=.084)$. The $\mathrm{HZ}$ vaccination rates did not differ significantly among the sites where patients receive primary care (Table 3 ).

Both patient education level and race independently correlated strongly with vaccination. In multivariate logistic regression analyses, patients with $\geq 12$ years of education were 4.6 times more likely (95\% CI, 1.3-16.2) to report receiving $\mathrm{HZ}$ vaccination and whites were 3.4 times more likely (95\% CI, 1.03-11.1) to report receiving herpes $\mathrm{HZ}$ vaccination when controlling for the effects of age, sex, having seen shingles before, and other demographic factors. Because of the potential for an interaction between race and education, we completed race-specific regression analyses. They demonstrated no association of education level with vaccination among AAs, which likely is due to a small sample of the vaccinated individuals in the AA group. However, there was a significant association between education level and $\mathrm{HZ}$ vaccination among whites when controlling for the effects of age, sex, and having seen shingles before. We also completed logistic regression modeling using an interaction term for race and education, which again demonstrated that whites with a high school education or higher were approximately 6.7 times more likely (95\% CI, 1.4-31.0) to receive the HZ vaccination compared with AAs with less than a high school education (Table 3).

Even among patients who were not vaccinated for HZ, those who had seen shingles in other people were more likely to be interested in receiving the $\mathrm{HZ}$ vaccine compared with those who had never seen someone develop shingles (66\% vs $47 \%$; $P<.001)$. Similarly, those people who had a personal history of shingles were more likely to be interested in getting the vaccine compared with

Table 2. Self-Reported Prevalence of Herpes Zoster, Witnessing Others With Zoster, Awareness of Vaccine, and Vaccination Rate by Race*

\begin{tabular}{|c|c|c|c|c|}
\hline & White $(\mathrm{n}=191)$ & African Americans $(\mathrm{n}=198)$ & Odds Ratio (95\% CI) & $P$ \\
\hline Reported having had shingles & $32(17)$ & $16(8)$ & $2.3(1.2$ to 4.3$)$ & .013 \\
\hline Had seen someone with shingles & $138(72)$ & $70(35)$ & $4.8(3.1$ to 7.3$)$ & $<.001$ \\
\hline Aware that the zoster vaccine is recommended & $81(42)$ & $32(16)$ & $3.8(2.4-6.1)$ & $<.001$ \\
\hline Had received zoster vaccine & $26(14)$ & $4(2)$ & $7.6(2.6-22.3)$ & $<.001$ \\
\hline
\end{tabular}

Values provided as $\mathrm{n}(\%)$ unless otherwise indicated.

*Data for only whites and African Americans are included here because of the small samples of other races. 
Table 3. Factors Associated With Herpes Zoster Vaccination

\begin{tabular}{|c|c|c|c|c|}
\hline & $\begin{array}{c}\text { All } \\
(\mathrm{N}=403)\end{array}$ & $\begin{array}{l}\text { HZ Vaccination } \\
\quad(\mathrm{n}=31)\end{array}$ & $\begin{array}{l}\text { No HZ Vaccination } \\
\quad(\mathrm{n}=372)\end{array}$ & $\begin{array}{l}\text { Odds Ratio } \\
(95 \% \mathrm{CI})\end{array}$ \\
\hline Age (years) & 72.8 & $74.4 \pm 9.3$ & $72.6 \pm 8.9$ & \\
\hline $60-64$ & $89(22.1)$ & $5(16.1)$ & $84(22.5)$ & $1.0^{*}$ \\
\hline$\geq 65$ & $312(77.4)$ & $26(83.8)$ & $286(76.8)$ & $1.53(0.57-4.1)$ \\
\hline \multicolumn{5}{|l|}{ Race, n (\%) } \\
\hline African American & $198(49)$ & $4(2)$ & $194(98)$ & $1.0^{*}$ \\
\hline White & $191(47)$ & $26(14)$ & $165(86)$ & $3.4(1.03-11.1) \dagger$ \\
\hline Education (years) & $11 \pm 3.2$ & $13.7 \pm 3.0$ & $11 \pm 3.2$ & \\
\hline$<12$ & $160(39.7)$ & $3(2)$ & $157(98)$ & $1.0^{*}$ \\
\hline$\geq 12$ & $215(53.3)$ & $23(11)$ & $192(89)$ & $4.6(1.3-6.2) \dagger$ \\
\hline \multicolumn{5}{|l|}{ Race and education interaction } \\
\hline AAs with $<$ high school & $104(25.8)$ & $2(2)$ & $102(98)$ & $1.0^{*}$ \\
\hline Whites with $\geq$ high school & $118(29.2)$ & $20(16.9)$ & $98(83)$ & $6.7(1.4-31.0) \dagger$ \\
\hline History of shingles & $48(11.9)$ & $4(12.9)$ & $44(11.8)$ & $1.1(0.37-3.27)$ \\
\hline Witnessed someone with shingles & $212(53)$ & $25(81)^{*}$ & $187(51)$ & $4.1(1.6-10.2) \dagger$ \\
\hline Received influenza vaccine in the past & $357(89)$ & $30(97)$ & $327(88)$ & $4.1(0.55-31.0)$ \\
\hline \multicolumn{5}{|l|}{ Clinic Site } \\
\hline Internal medicine clinic & $134(33)$ & $13(42)$ & $121(33)$ & $1.0^{*}$ \\
\hline Family medicine clinic & $133(33)$ & $6(19)$ & $127(34)$ & $0.44(0.16-1.19)$ \\
\hline Geriatric clinic & $134(33)$ & $12(39)$ & $122(33)$ & $0.92(0.4-2.1)$ \\
\hline
\end{tabular}

Values provided as $\mathrm{n}(\%)$. Values may not add to 100 percent due to some subjects declining to answer some parts of the survey. ${ }^{*}$ Reference group for comparison.

$\dagger$ Multivariate logistic regression analysis controlling for effects of age, sex, race, education, and having seen someone with shingles. $\mathrm{HZ}=$ Herpes Zoster; AAs = African Americans.

people who reported no history of shingles ( $76 \%$ vs $54 \% ; P=.006)$.

Table 4 shows reasons for receiving or not receiving the vaccine as cited by the vaccinated and unvaccinated participants. Overall, $13.7 \%$ of all study subjects reported having any communication with their medical providers regarding the $\mathrm{HZ}$ vaccine. Of the vaccinated participants, $48 \%$ individuals reported that the vaccine had been recommended by their physicians and 32\% cited the media. Of the 370 participants who reported not being vaccinated, $70 \%$ had never heard about it, $11 \%$ had not had it recommended by their physician, and $11 \%$ felt they did not need it. After completing the survey, $59 \%$ of those who had not been vaccinated were interested in receiving the vaccine.

\section{Discussion}

This study investigated the $\mathrm{HZ}$ vaccination rate among the target population during a sampling of patients at 3 different university primary care clinics and found that the vaccination rate overall was low. Furthermore, we found a racial disparity in the percentage of patients vaccinated that persisted in multivariate analyses, with AAs being far less likely to report vaccination. We postulate that the lower $\mathrm{HZ}$ vaccination rate among AAs is partially the result of the lower self-reported prevalence of $\mathrm{HZ}$ and the lower rate of witnessing friends/family with $\mathrm{HZ}$-all of which may influence perceived risk and therefore interest in vaccination.

In support of our hypothesis regarding the potential contribution of disease prevalence to racial differences in vaccination rate, we found a significant difference in the prevalence of self-reported HZ between whites and AAs. Our study showed whites were more than twice as likely as AAs to report having had $\mathrm{HZ}$ and more than 4 times more likely of having seen someone else with $\mathrm{HZ}$. Schmader et $\mathrm{a}^{5,7}$ reported an even larger difference in $\mathrm{HZ}$ prevalence between the 2 races $(16.1 \%$ white, $4.5 \% \mathrm{AA})$. The reasons for this reported differences in the disease prevalence by race remain unclear but likely are multifactorial, including potential biologic differences, the recognition and reporting of the disease, and the frequency and intensity of medical interactions. These comparisons 
Table 4. Factors Impacting Decisions For or Against Receiving the Herpes Zoster (HZ) Vaccine

\begin{tabular}{lr}
\hline Reasons cited for getting the HZ vaccine $(\mathrm{n}=31)$ & $15(48)$ \\
Recommended by health care provider & $10(32)$ \\
Recommended by media/ads & $6(19)$ \\
Recommended by friends & $5(16)$ \\
Recommended by family & $3(10)$ \\
Other & \\
Reasons cited for not getting the HZ vaccine & \\
$\quad$ (n = 370) & $258(70)$ \\
Have not heard about it & $42(11)$ \\
Do not think I will develop shingles & $40(11)$ \\
Physician did not recommend it & $29(8)$ \\
Afraid of the side effects & $15(4)$ \\
Financial reasons & $14(4)$ \\
Do not believe in vaccines in general & $5(1)$ \\
Have a weak immune system and cannot receive & \\
live virus vaccines & $4(1)$ \\
Allergic to the zoster vaccine & $3(0.8)$ \\
Do not think shingles will cause significant or & \\
lasting illness & $2(0.5)$ \\
Do not think the vaccine works & 0 \\
Someone told me not to get the vaccine &
\end{tabular}

Values provided as $\mathrm{n}(\%)$. More than one response was accepted for each question.

of disease prevalence unfortunately are limited in that both studies used self-report to determine the prevalence of HZ. However, our survey showed that witnessing someone with $\mathrm{HZ}$ has a large impact on an individual's vaccination rate and the desire to be vaccinated.

In the study by Oxman et $\mathrm{al},{ }^{2}$ cited by the Advisory Committee on Immunization Practices, the sample of 38,546 subjects was $95 \%$ white and only $2 \% \mathrm{AA}$, with no racial comparison of $\mathrm{HZ}$ vaccine efficacy reported. Although the current Centers for Disease Control and Prevention recommendations are to vaccinate all immunocompetent persons older than age 60 regardless of race, more research of various races is required to determine whether vaccination should be recommended for all races based on incidence, efficacy, and cost-effectiveness in various groups. One retrospective study found that the zoster vaccine use was linked with a $66 \%$ decreased risk of zoster in AAs, but a prospective study is needed to confirm this finding and to make conclusions regarding the vaccine's cost-effectiveness in this population. ${ }^{17}$

We demonstrated that education also was independently associated with vaccination rates. When examined further in race-specific regression analy- ses, the significant relationship of education to vaccination was identified for white patients only. The lack of a significant relationship among AAs may have been due to the small number of AAs who reported vaccination and the lower variation in education levels, therefore limiting our ability to find a significant association. The results imply that more attention should be given to people with lower education level to inform them of the vaccination recommendation.

The most common reason given in our study for not having the $\mathrm{HZ}$ vaccination was that patients were not aware that it was recommended; this was cited by $70 \%$ of the surveyed patients who have not had $\mathrm{HZ}$ vaccine. Only $13.7 \%$ of all study subjects reported having any communication with their medical providers regarding the $\mathrm{HZ}$ vaccine. This suggests the need to educate both medical providers and patients regarding $\mathrm{HZ}$ vaccine recommendations. The importance of awareness was underscored by our finding that the majority of patients who were not vaccinated responded positively when asked whether they would be interested in receiving the vaccine after learning more about $\mathrm{HZ}$ through the survey process. From this we postulate that being aware that the vaccine exists and having medical providers recommend it may greatly improve $\mathrm{HZ}$ vaccination rates. Eight percent of the patients cited potential adverse effects as a reason to avoid the zoster vaccine. However, the reported rates of serious adverse effects of the zoster vaccine are low $(1.4 \%)$; the rate was not different from patients who received placebo. ${ }^{18}$ This finding indicates the need for better discussion with and education of patients.

The cost of $\mathrm{HZ}$ vaccine previously had been cited as a major obstacle to vaccination. ${ }^{15}$ Medicare now covers the cost of the vaccine for eligible individuals. In this study of patients with mostly Medicare coverage, financial factors were cited by only a small number. We also showed that the rate of vaccination was not different between people in age 60 to 64 group versus 65 and older. These age groups were selected for analysis because patients aged 65 and older traditionally will have Medicare coverage. However, even with Medicare coverage, some patients may have co-payments or fall in the coverage gap, known as the "donut hole," that cause financial burden. For uninsured patients or for those with insurance plans that do not fully 
cover the cost of the vaccine, expense may be a more important factor. ${ }^{19}$

Although not specifically studied in our survey, additional potential barriers to $\mathrm{HZ}$ vaccination exist. One example is the complexity of the vaccine administration. ${ }^{14}$ The vaccine must be stored at $-15^{\circ} \mathrm{C}$ $\left(+5^{\circ} \mathrm{F}\right)$, and it must be administered within 30 minutes of thawing. ${ }^{20}$ This makes it inconvenient to administer in practices that do not have the vaccine in the building. When this is the case, systems-based plans need to be implemented to simplify the acquisition and administration of the vaccine. For the 3 clinics studied here, there was one central pharmacy located in the family medicine center that dispensed the $\mathrm{HZ}$ vaccine. Patients picked up the vaccine and had to bring it back to their respective clinic within 30 minutes for administration. As an alternative, they took their zoster vaccine prescription to private pharmacies off-site for dispensing and administration.

At the time of our study, the electronic health records at the study sites did not keep track of the zoster vaccination status because of the relatively recent vaccine recommendations. A simple reminder for preventive services regarding the zoster vaccine in electronic health records could potentially increase the provider's awareness of and willingness to discuss the zoster vaccine with patients and therefore increase the overall vaccination rate.

\section{Limitations}

The limitations of our study include that all data were self-reported. Though self-report of $\mathrm{HZ}$ has been shown to be accurate, it is difficult to determine the accuracy of patients' reports of witnessing someone with $\mathrm{HZ}$ or of having received various vaccines. ${ }^{21}$ Recall bias cannot be ruled out. Although the rate of participation of patients in the survey was extremely high subjectively, the study team did not keep a record of the few patients who refused to participate and their demographic characteristics. This may introduce a selection bias.

Our study comprised mostly AAs and whites with few subjects of other races; this limited racial composition prohibited meaningful analysis of $\mathrm{HZ}$ and vaccination rates among other races. This relatively small sample size limits our power to determine accurate prevalence rates.

This study used a convenience sample of patients presenting to university-based primary care practices and may not be a representative sample of our overall community population. It is possible that the $\mathrm{HZ}$ vaccination rate in our study population is higher than in the general community because the subjects already were being exposed to health care professionals by coming to a primary care clinic. In the general community, there may be some people who do not have a primary care provider or access to health care and therefore are less likely to have received the $\mathrm{HZ}$ vaccine.

Clinicians' clinical practice patterns or knowledge of the current $\mathrm{HZ}$ vaccination recommendations were not assessed in this study. This limits our ability to suggest that low vaccination rates may have resulted from providers not having care delivery systems or provider education that facilitate vaccination in response to published guidelines. Future studies would need to incorporate ways to both inform and encourage practitioners to prescribe the vaccine for their appropriate patients.

\section{Conclusions}

$\mathrm{HZ}$ vaccination rates are low, as is patients' awareness that the $\mathrm{HZ}$ vaccine is recommended for all people older than age 60. Large differences in the vaccination rates exist between $\mathrm{AAs}$ and whites, and potential reasons for the differences are likely multifactorial. Witnessing someone having $\mathrm{HZ}$ and having a higher educational level are strongly related to $H Z$ vaccination status. A physician's recommendation is the reason to get vaccinated that is most commonly cited by patients. Not being aware of the vaccine and the recommendation was reason most commonly cited by patients who had not received the vaccine, and most of these people wanted the vaccine once they knew about it. Future research should explore the incidence of shingles and the efficacy of vaccination in AAs and other minority races because some populations have a lower incidence of the disease and the cost-effectiveness of the vaccine in these groups is not known. To achieve higher HZ vaccination rates, public awareness, education, and redesign of the health care delivery system should be explored.

We acknowledge the following individuals: Katherine Rickett, MSLS, MSEd, NCC, from the East Carolina University Laupus Library, assisted with organizing and formatting the references. James Powell, MD, from East Carolina University Internal Medicine Clinic, and Robert Newman, MD, from East Carolina University Family Medicine Clinic, facilitated with conducting this research study in the clinics. 


\section{References}

1. Arvin AM. Varicella-zoster virus. Clin Microbiol Rev 1996;9:361-81.

2. Oxman MN, Levin MJ, Johnson GR, et al. A vaccine to prevent herpes zoster and postherpetic neuralgia in older adults. N Engl J Med 2005;352:2271-84.

3. Jumaan AO, Yu O, Jackson LA, et al. Incidence of herpes zoster, before and after varicella-vaccinationassociated decreases in the incidence of varicella, 1992-2002. J Infect Dis 2005;191:2002-7.

4. Insinga RP, Itzler RF, Pellissier JM, et al. The incidence of herpes zoster in a United States administrative database. J Gen Intern Med 2005;20:748-53.

5. Schmader K, George LK, Burchett BM, et al. Racial differences in the occurrence of herpes zoster. J Infect Dis 1995;171:701-4.

6. Chaves SS, Santibanez TA, Gargiullo P, et al. Chickenpox exposure and herpes zoster disease incidence in older adults in the U.S. Public Health Rep 2007; 122:155-9.

7. Schmader K, George LK, Burchett BM, et al. Race and stress in the incidence of herpes zoster in older adults. J Am Geriatr Soc 1998;46:973-7.

8. Katz J, Cooper EM, Walther RR, et al. Acute pain in herpes zoster and its impact on health-related quality of life. Clin Infect Dis 2004;39:342-8.

9. Yawn BP, Saddier P, Wollan PC, et al. A populationbased study of the incidence and complication rates of herpes zoster before zoster vaccine introduction. Mayo Clin Proc 2007;82:1341-9.

10. Harpaz R, Ortega-Sanchez IR, Seward JF; Advisory Committee on Immunization Practices (ACIP) Centers for Disease Control and Prevention (CDC). Prevention of herpes zoster: recommendations of the Advisory Committee on Immunization Practices (ACIP). MMWR Recomm Rep 2008;57: 1-30; quiz CE32-34.

11. Sun W, US Food and Drug Administration. Approval letter - Zostavax [letter online]. March 24, 2011. Available at: www.fda.gov/biologicsbloodvaccines/ vaccines/approvedproducts/ucm248608.htm. Accessed September 12, 2011.
12. Hensley S, NPR. FDA gives OK to broader use of shingles vaccine [article online]. Available at: www. npr.org/blogs/health/2011/03/25/134849776/zostavaxfda-gives-ok-to-broader-use-of-shingles-vaccine. Accessed September 12, 2011.

13. Lu PJ, Euler GL, Jumaan AO, et al. Herpes zoster vaccination among adults aged 60 years or older in the United States, 2007: uptake of the first new vaccine to target seniors. Vaccine 2009;27:882-7.

14. Wolf F, Michaud K. Rates and predictors of influenza, pneumococcal, and herpes zoster vaccination in adult patients with rheumatic diseases. Presented at the ACR/AHRP Scientific Meeting, October 1721, 2009, Philadelphia, PA. Available at: http://acr. confex.com/acr/2009/webprogram/Paper10757.html. Accessed March 1, 2012.

15. Hurley LP, Lindley MC, Harpaz R, et al. Barriers to the use of herpes zoster vaccine. Ann Intern Med 2010;152:555-60.

16. Herpes zoster vaccine (Zostavax). Med Lett Drugs Ther 2006;48:73-4.

17. Tseng H, Smith N, Harpez R. Herpes zoster vaccine in older adults and the risk of subsequent herpes zoster disease. JAMA 2011;305:160-16.

18. Simberkoff M, Arbeit R, Johnson G. Safety of Herpes Zoster Vaccine in the Shingles Prevention Study: a randomized trial. Ann Intern Med 2010; 152:545-54.

19. The Harvard Medical School Family Health Guide. The shingles vaccine: why hasn't it caught on? 2007. Available at: www.health.harvard.edu/fhg/updates/ The-shingles-vaccine.shtml. Accessed September 12, 2011.

20. Zostavax: full prescribing information. June 2011. Whitehouse Station, NJ: Merck \& Co, Inc. Available at: www.merck.com/product/usa/pi_circulars/ z/zostavax/zostavax_pi2.pdf. Accessed September 12, 2011.

21. Schmader K, George LK, Newton R, et al. The accuracy of self-report of herpes zoster. J Clin Epidemiol 1994;47:1271-6. 